\title{
The Relationship of Loneliness to End-of-Life Experience in Older Americans: A Cohort Study
}

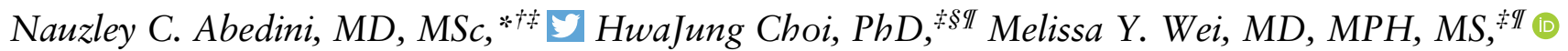 \\ Kenneth M. Langa, MD, PhD, ${ }^{\ddagger \$ l l}$ and Vineet Chopra, MD, MSc $c^{\dagger \neq l l}$
}

OBJECTIVES: Little is known about the relationship between loneliness and end-of-life (EOL) experience including symptom burden, intensity of care, and advance care planning among older adults.

DESIGN: Secondary analysis of the Health and Retirement Study (HRS).

SETTING: Population based.

PARTICIPANTS: Decedents older than 50 years who died between 2004 and 2014 ( $\mathrm{n}=8700)$. Exclusions included those who were ineligible for surveys assessing loneliness $(\mathrm{n}=2932)$ or had missing or incomplete loneliness or symptom data $(\mathrm{n}=2872)$.

MEASUREMENTS: Individuals were characterized as lonely based on responses to the three-item Revised University of California, Los Angeles Loneliness Scale in the most recent HRS survey before death. Outcomes were proxy reports of total EOL symptom burden, intensity of EOL care (eg, late hospice enrollment, place of death, hospitalizations, use of life support), and advance care planning. Results were expressed as adjusted odds ratios (aORs) with 95\% confidence intervals (CIs).

RESULTS: One-third of 2896 decedents $(\mathrm{n}=942)$ were lonely. After adjusting for demographics, socioeconomic

From the *National Clinician Scholars Program, University of Michigan, Ann Arbor, Michigan; 'Division of Hospital Medicine, University of Michigan, Ann Arbor, Michigan; "Institute for Healthcare Policy and Innovation, University of Michigan, Ann Arbor, Michigan; ${ }^{\circledR}$ Institute for Social Research, University of Michigan, Ann Arbor, Michigan; "Division of General Internal Medicine, University of Michigan, Ann Arbor, Michigan; and the "Veterans Affairs Center for Clinical Management Research, Ann Arbor, Michigan.

Address correspondence to Nauzley Abedini, MD, MSc, Institute for Healthcare Policy and Innovation, University of Michigan, North Campus Research Complex, 2800 Plymouth Road, Building 14, G100-17, Ann Arbor, MI 48109-2800. E-mail: nauzley@umich.edu. Twitter: @NauzleyAbedini

Prior presentations: Society of Hospital Medicine Meeting, National Harbor, MD, March 2019; Hamolsky Junior Faculty Scientific Award Presentation at the Society of General Internal Medicine Meeting, Washington, DC, May 2019.

DOI: 10.1111 /jgs. 16354 status, multimorbidity, depressive symptoms, family and friends, and social support, loneliness was independently associated with increased total symptom burden at EOL $(ß=.13 ; P=.004)$. Compared with nonlonely individuals, lonely decedents were more likely to use life support in the last 2 years of life $(35.5 \%$ vs $29.4 \%$; aOR $=1.36 ; 95 \%$ $\mathrm{CI}=1.08-1.71)$ and more likely to die in a nursing home $(18.4 \%$ vs $14.2 \%$; aOR $=1.78 ; 95 \% \mathrm{CI}=1.30-2.42)$. No significant differences in other measures of intense care (late hospice enrollment, number of hospitalizations, or dialysis use) or likelihood of advance care planning were observed.

CONCLUSION: Lonely older people may be burdened by more symptoms and may be exposed to more intense EOL care compared with nonlonely people. Interventions aiming to screen for, prevent, and mitigate loneliness during the vulnerable EOL period are necessary. J Am Geriatr Soc 68:10641071, 2020.

Key words: end of life; loneliness; aggressive care; advance care planning; symptoms

L oneliness, the subjective feeling of isolation, lack of belonging, or lack of companionship, ${ }^{1}$ afflicts approximately $40 \%$ of older adults ${ }^{2,3}$ and is associated with poor health outcomes such as depression, ${ }^{4}$ accelerated functional ${ }^{2}$ and cognitive decline, ${ }^{5}$ and early mortality. ${ }^{1,6}$ As such, loneliness and social isolation, an objective measure of social networks and connection, are deemed important social determinants of health worthy of routine screening by the National Academies and the Centers for Medicare \& Medicaid Services. ${ }^{7,8}$ Poor health outcomes related to loneliness are frequently linked with poor quality of life in older adults ${ }^{9}$ and may also be associated with suffering at end of life (EOL). Role transitions, shrinking social networks, and mounting multimorbidity are common at EOL and can increase both the risk of loneliness and its downstream health consequences. ${ }^{10}$ 
Important markers of EOL experience and care quality include symptom burden, exposure to aggressive or intense EOL care, and presence of advance care planning. ${ }^{11}$ Yet little is known about how loneliness affects these markers. What is known is that older adults are prone to high symptom burden and experience a peak in symptom burden in the months preceding death, ${ }^{12}$ and they experience burdensome symptoms at EOL more than ever before. ${ }^{12-15}$ It is plausible that the increased disease burden experienced by lonely older adults may contribute to higher symptom burden at EOL, but studies assessing this relationship are lacking. Similarly, older adults are frequently exposed to intense or aggressive EOL care despite evidence that it can cause suffering for patients and families. ${ }^{16,17}$ Whether this construct holds true for lonely adults, and how it relates to use of invasive interventions such as life support, hemodialysis, hospitalizations, and use of hospice, remains unclear. Furthermore, given that loneliness affects an individual's ability to connect with others,$^{18}$ it may potentially affect their ability to participate in advance care planning. Understanding the relationship between loneliness and markers of EOL experience is important given that loneliness may be modifiable. ${ }^{1}$ Mitigating EOL loneliness may help improve EOL experience in this vulnerable population.

Using a cohort of older decedents from 2004 to 2014 in the Health and Retirement Study (HRS), we examined the impact of subjective loneliness on EOL experience as measured by symptom burden, intensity of care, and advance care planning at EOL. We hypothesized that loneliness would be an independent predictor of increased symptom burden, increased exposure to intense EOL care, and decreased use of advance care planning.

\section{METHODS}

\section{Data Source and Study Participants}

The HRS, a nationally representative longitudinal survey of adults 51 years of age and older, is conducted every 2 years ${ }^{19}$ and has a similar sample mortality rate as the agematched US population. ${ }^{20}$ Using publicly available data from the HRS, we examined participants who died between 2004 and 2014. After an HRS participant dies, proxy informants (often a spouse/partner or other family member) familiar with the decedent's health, finances, and social interactions complete an exit interview that includes information about the participant's EOL experience (ie, symptoms, advance care planning, and healthcare utilization and exposure to intense EOL care measures).

Approximately one-half of HRS participants are eligible for a Psychosocial and Lifestyle Questionnaire containing a three-item loneliness scale during alternating waves (every 4 years). ${ }^{21}$ We selected 2004 as the initial starting point for the sample because it was the first year in which the Psychosocial and Lifestyle Questionnaire was administered.

Of the 8700 decedents in the HRS from 2004 to 2014, 2896 decedents met all inclusion criteria and were included in our final sample. Others were excluded due to lack of exit interviews ( $\mathrm{n}=961$ ); lack of loneliness data due to Psychosocial and Lifestyle Questionnaire ineligibility $(\mathrm{n}=2932)$, nonresponse $(\mathrm{n}=1555)$ or partial response $(\mathrm{n}=187)$; or

\section{0 decedents in HRS from 2004-2014}

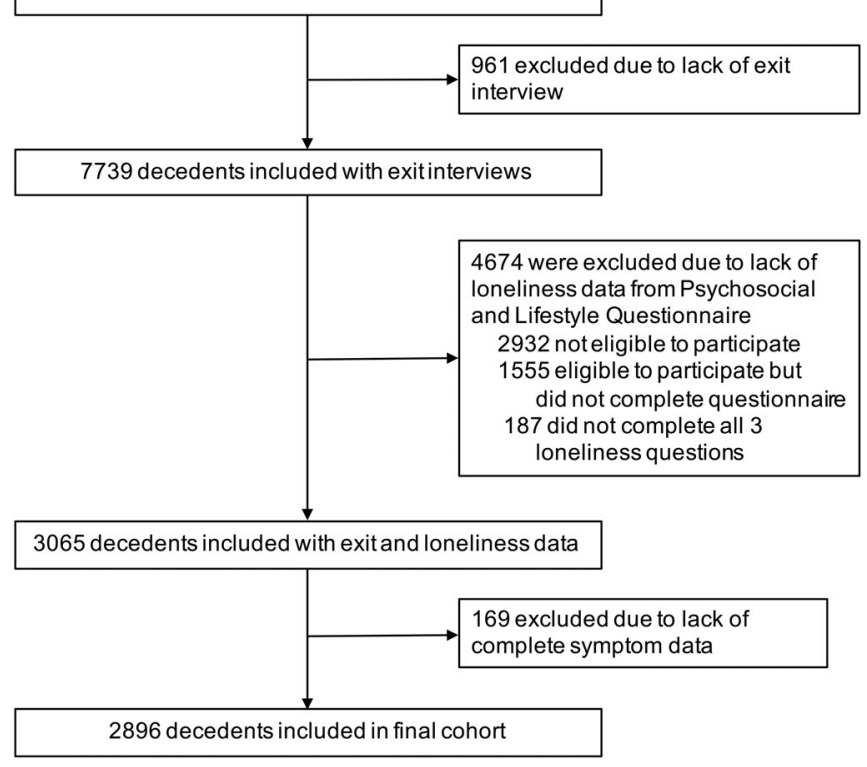

Figure 1. Construction of the Health and Retirement Study (HRS) decedent cohort, 2004-2014.

incomplete EOL symptom data $(\mathrm{n}=169)$ (Figure 1). The HRS complies with the requirements of the University of Michigan's institutional review board.

\section{Variables}

\section{Predictor Variable: Loneliness}

We used the most recent loneliness data from the Psychosocial and Lifestyle Questionnaire before death to approximate loneliness status close to death. Consistent with prior studies $^{2,22}$ and recommendations from the HRS, ${ }^{21}$ we constructed a dichotomous loneliness measure using the validated three-item Revised University of California, Los Angeles (UCLA) Loneliness Scale (R-UCLA). ${ }^{23}$ The three items include whether the participant feels left out, feels isolated, and/or lacks companionship (Cronbach's $\alpha=.81$ ). The participant is asked to rate each as "Often," "Some of the time," or "Hardly ever or never." The responses were reverse coded on a 1 to 3 scale, and an average score was calculated for all three components. ${ }^{21}$ We classified individuals with an average score of 1.9 or less as "nonlonely" and those with an average score of 2.0 or more as "lonely" that corresponded to previously established cut points for the dichotomous loneliness variable., ${ }^{2,22}$

\section{Primary Outcome: Total Symptom Burden}

HRS proxies report the presence or absence of specific symptoms for one or more months during the last year of the decedent's life: pain, difficulty breathing, severe fatigue, very little appetite, frequent vomiting, difficulty controlling limbs, periodic confusion, and difficulty awakening. Because the HRS does not contain so-called gold standard symptom scales (due to their length and time-intensive nature), ${ }^{24}$ we created a composite scale for total symptom burden in the last year of life. We used a composite scale because 
symptoms are often experienced in aggregate, with each symptom contributing variably to suffering. ${ }^{25} \mathrm{We}$ used principal-component factor analysis to construct the composite scale ${ }^{26}$ using an Eigenvalue threshold greater than 1.0 and factor loading greater than .3. All symptoms were included in the final factor that used predictive regression models to assign weights to each individual symptom (Cronbach's $\alpha=.65$ ).

\section{Secondary Outcomes}

Secondary outcomes were assessed individually. For intensity of EOL care, we used these outcome variables traditionally associated with low-value intense EOL care: ${ }^{27,28}$ proportion spending three or fewer days in hospice (late hospice referral); place of death; use of intensive care units (ICUs), hospitals, and nursing homes; and use of life support and dialysis in the last 2 years of life.

For advance care planning, markers included whether the decedent had (1) discussed EOL care preferences with next of kin, (2) assigned a durable power of attorney, (3) written EOL instructions, or (4) participated in EOL decisions before death. ${ }^{28}$

\section{Covariates}

We used the most recent HRS survey before death corresponding with the wave in which the loneliness questions were administered. We included these four covariates

Table 1. Characteristics of Lonely and Nonlonely Decedents, 2004-2014 $(\mathrm{n}=2986)^{\mathrm{a}}$

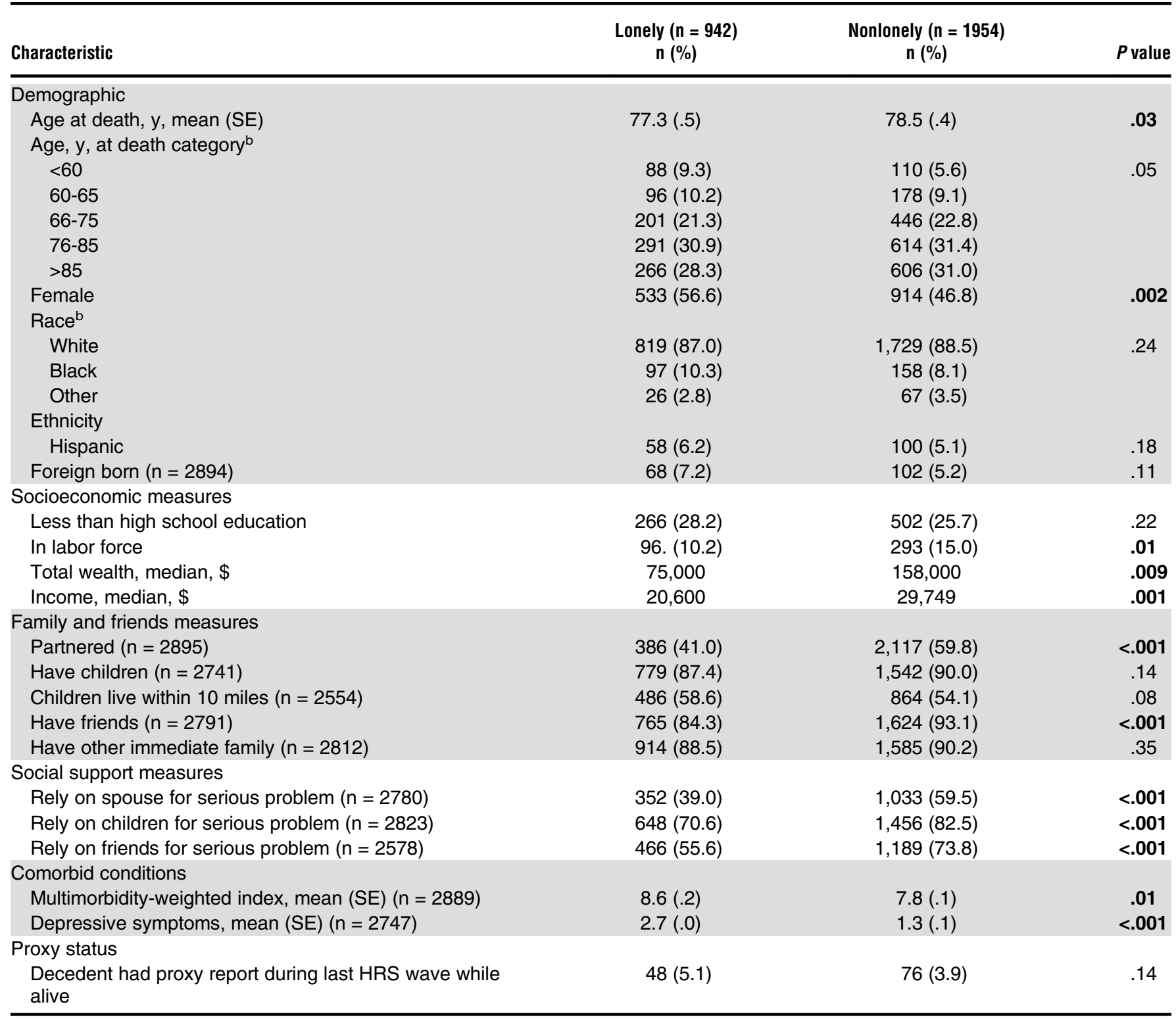

Note: Boldface indicates a statistically significant value $(P<.05)$.

Abbreviations: HRS, Health and Retirement Study; SE, standard error.

${ }^{a} \mathrm{n}=2896$ for each characteristic unless otherwise defined.

bercentages do not sum to $100 \%$ due to rounding. 
known to influence loneliness and/or our outcomes of interest $^{1}$ in multivariable models:

1. Demographic and socioeconomic measures (age at death, sex, ethnicity, education, income, and employment status);

2. Multimorbidity (ie, multiple chronic conditions), via a validated multimorbidity-weighted index (MWI) computed from participant self-report of 15 physiciandiagnosed conditions. ${ }^{29,30}$ Because the MWI is strongly associated with current and future physical functioning, activities of daily living, instrumental activities of daily living, and cognitive decline, ${ }^{27,29}$ these variables were omitted to prevent multicollinearity;

3. Depressive symptoms, determined using the Center for Epidemiologic Studies Depression Scale (CES-D) selfreport of symptoms. ${ }^{31}$ The HRS uses an eight-item version of the CES-D that includes a question about whether the individual feels lonely. Given concerns for confounding with our predictor variable, this loneliness question was removed from the CES-D for our analysis; depressive symptoms were reported on a scale of 0 to 7 based on responses to the remaining CES-D questions (Cronbach's $\alpha=.75$ );

4. Family and friends (partner status, having children, having children living within 10 miles, having friends, and having any other immediate family), ${ }^{32}$ and social support (reliance on their spouse, children, or friends for serious problems). ${ }^{32}$

\section{Statistical Analysis}

We used population survey weights ${ }^{33}$ to account for the complex survey design of the HRS for all of our analyses including imputation models. We first summarized characteristics for participants who were "lonely" and "nonlonely," estimating differences using $\chi^{2}$ tests for categorical variables and $t$ tests for continuous variables. A two-tailed $P$ value $<.05$ was considered statistically significant. Next, we assessed the

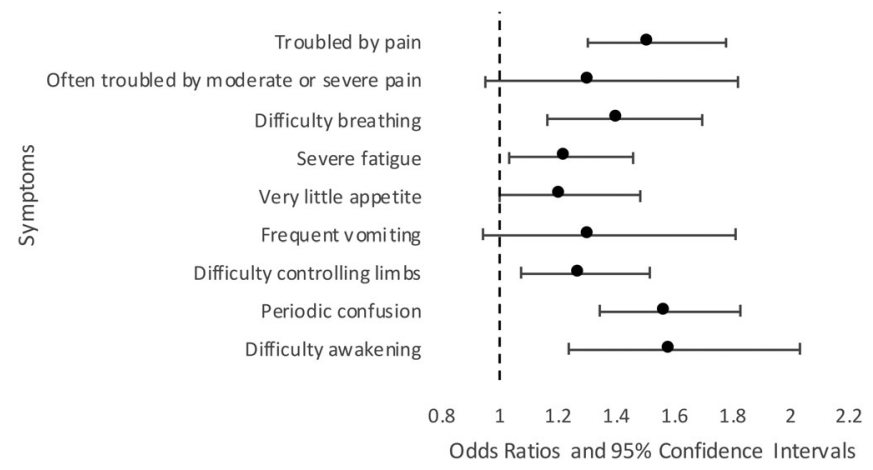

Figure 3. Unadjusted odds of symptoms experienced by lonely vs nonlonely older adults in the last year of life $(n=2896)$.

relationship between loneliness and individual symptoms using bivariate logistic regression. We then used both bivariate and multivariable regressions with ordinary least squares models to assess the relationship of loneliness to total symptom burden at EOL, adjusting for covariates. Our initial model adjusted for baseline covariates including demographic characteristics, socioeconomic status, and multimorbidity (model 1). We then added the following covariates to the baseline model sequentially to assess various confounders: depressive symptoms (model 2), family and friends (model 3), and social support (model 4). Some measures of family, friends, and social support were excluded because their baseline relationship with loneliness was not significant (Table 1). In the final model (model 5), we adjusted for all covariates in models 1 to 4 (Table S1).

Using the final adjusted model, we also examined the relationship of loneliness to intense EOL care and advance care planning. We used multivariable logistic regression for dichotomized outcomes and negative binomial regression for count measures.

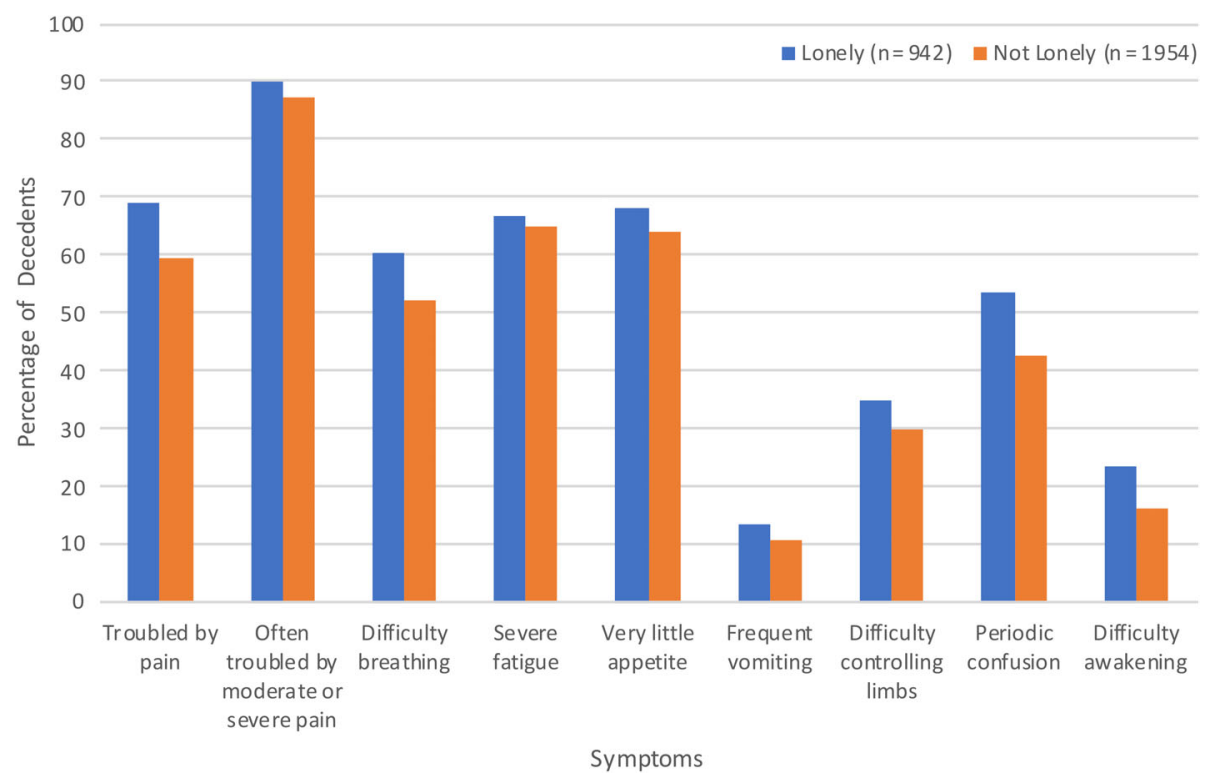

Figure 2. Proportion of lonely vs nonlonely older adults experiencing individual symptoms in the last year of life $(\mathrm{n}=2896)$. 


\section{Missing Data}

Approximately $10 \%$ of covariates in our baseline sample had missing data. Four covariates (depressive symptoms, relying on spouse, having friends, and relying on children) accounted for roughly $9 \%$ of missing data. Missing covariates were imputed using chained equations with 10 iterations. ${ }^{34}$

We conducted analyses with Stata v.15.1 IC (StataCorp, College Station, TX). All analyses were performed by N.A. and H.C.

\section{Evaluation for Multicollinearity}

The variance inflation factor (VIF) was calculated for the covariates in our final adjusted imputed model. Multicollinearity was mild based on a maximum VIF of 1.45 for all covariates.

\section{Additional Analyses}

To assess for selection bias, we evaluated the relationship between loneliness and the likelihood of having an exit interview in our baseline decedent cohort. Lonely decedents had similar odds of having an exit interview (odds ratio $=.93 ; P=.53$ ).

To account for possible variance in loneliness over time, we introduced a time-varying covariate representing the duration of time between completion of the loneliness scale and death. Similarly, to assess for recall bias, we introduced another time-varying covariate representing the duration of time between death and the exit interview. Additionally, HRS participants who were unable (due to physical or cognitive deficits) to provide self-report during the main survey may have had proxy reports of covariates that may be less reliable than self-report. Hence we included a covariate related to proxy status in the most recent HRS survey before death. Point estimates adjusting for each of these covariates were similar for symptoms and measures of intense care. Thus these duration covariates and proxy status were excluded from the final regression models.

Finally, to assess the influence of missing data, all multivariable analyses were performed with and without imputed data (Tables S2 and S3) with no differences in estimates for both.

\section{RESULTS}

\section{Participant Characteristics}

Our final sample consisted of 2896 decedents (Figure 1). The mean duration of time from completion of the Psychosocial and Lifestyle Questionnaire to death was 2.7 years \pm 1.8 years and was not significantly different for lonely vs nonlonely individuals $(P=.72)$. The mean duration of time from death to exit interview completion was 1.0 years \pm .7 year and was not different for lonely vs nonlonely individuals $(P=.78)$.

Table 1 summarizes the decedent characteristics in the 2004 to 2014 HRS cohort. Of the 2896 decedents, $942(34 \%)$ were lonely in the final sample. At the time of death, lonely decedents were younger (mean age $=77.3 \mathrm{y}$ vs $78.5 \mathrm{y}$ in nonlonely individuals; $P=.03$ ) and more often female $(P=.002)$. Similarly, they were less likely to be partnered or have friends than those that were nonlonely

Table 2. Relationship of Loneliness to Measures of Intense Care, Healthcare Utilization, and Advance Care Planning at End of Life

\begin{tabular}{|c|c|c|c|c|c|c|}
\hline Outcome & $\begin{array}{l}\text { Lonely } \\
\mathrm{n}(\%)\end{array}$ & $\begin{array}{l}\text { Nonlonely } \\
\mathrm{n}(\%)\end{array}$ & $\begin{array}{l}\text { Unadjusted OR } \\
\quad(95 \% \text { Cl) }\end{array}$ & $\begin{array}{c}P \\
\text { value }\end{array}$ & $\begin{array}{l}\text { aORa ratio } \\
(95 \% \mathrm{Cl})\end{array}$ & $\begin{array}{c}P \\
\text { value }\end{array}$ \\
\hline \multicolumn{7}{|l|}{ Measures of intense EOL care } \\
\hline$\leq 3 \mathrm{~d}$ in hospice $(n=2484)$ & $665(82.7)$ & $1,297(83.6)$ & $.94(.75-1.17)$ & .57 & $.93(.73-1.19)$ & .57 \\
\hline \multicolumn{7}{|l|}{ Death location $(n=2481)$} \\
\hline Home & $242(30.0)$ & 557 (35.9) & Ref & Ref & Ref & Ref \\
\hline Hospital & $278(34.5)$ & $519(33.5)$ & $1.23(.99-1.52)$ & .06 & $1.28(1.01-1.62)$ & .04 \\
\hline Nursing home & $148(18.4)$ & $220(14.2)$ & $1.56(1.23-1.96)$ & $<.001$ & $1.78(1.30-2.42)$ & $<.001$ \\
\hline Spent time in ICU in last $2 y$ of life $(n=904)$ & $151(51.5)$ & $275(48.7)$ & $1.12(.90-1.39)$ & .32 & $1.09(.84-1.39)$ & .52 \\
\hline $\begin{array}{l}\text { Used life support equipment in last } 2 y \text { of life } \\
(n=1949)\end{array}$ & $225(35.5)$ & $358(29.4)$ & $1.32(1.08-1.62)$ & .01 & $1.36(1.08-1.71)$ & .01 \\
\hline Dialysis in last 2 y of life $(n=1968)$ & $43(6.8)$ & $101(8.2)$ & $.81(.52-1.27)$ & .35 & $.71(.43-1.17)$ & .17 \\
\hline \multicolumn{7}{|l|}{ Other healthcare utilization in last $2 \mathrm{y}$ of life } \\
\hline Hospital nights, IRR $(\mathrm{n}=1740)$ & $\ldots$ & $\ldots$ & $1.18(.81-1.71)$ & .37 & $.95(.66-1.36)$ & .78 \\
\hline Nursing home nights, IRR $(\mathrm{n}=365)$ & & $\ldots$ & $.69(.38-1.27)$ & .22 & $.90(.50-1.60)$ & .71 \\
\hline \multicolumn{7}{|l|}{ Advance care planning } \\
\hline Discussed EOL care $(n=2460)$ & $524(65.6)$ & $985(64.1)$ & $1.07(.86-1.33)$ & .54 & $1.08(.87-1.35)$ & .46 \\
\hline $\begin{array}{l}\text { Assigned durable power of attorney } \\
(\mathrm{n}=2417)\end{array}$ & $521(66.3)$ & $971(64.3)$ & $1.09(.90-1.32)$ & .36 & $1.13(.88-1.46)$ & .34 \\
\hline Written EOL care instructions $(n=2440)$ & $404(51.0)$ & $828(54.3)$ & $.88(.71-1.08)$ & .21 & $.95(.78-1.15)$ & .57 \\
\hline $\begin{array}{l}\text { Subject participated in EOL decisions } \\
(n=1051)\end{array}$ & $121(35.3)$ & $260(39.6)$ & $.83(.61-1.14)$ & .24 & $.77(.55-1.06)$ & .10 \\
\hline
\end{tabular}

Note: Boldface indicates a statistically significant value $(P<.05)$.

Abbreviations: aOR, adjusted odds ratio; CI, confidence interval; EOL, end of life; ICU, intensive care unit; IRR, incidence risk ratio; OR, odds ratio.

${ }^{a}$ Adjusted for race, age, sex, education, total income, multimorbidity-weighted index, depressive symptoms, family and friends (partner status, has friends), and social support (relies on spouse, relies on children, relies on friends). 
$(P<.001$ for both). Lonely decedents had a significantly higher mean MWI index score (8.6 vs $7.8 ; P=.01)$, suggesting higher overall disease burden and worse functioning. They were also significantly less likely to rely on their spouse, children, or friends for serious problems $(P<.001$ for all outcomes $)$. Lonely decedents were more likely to have more depressive symptoms on average $(P<.001)$. Finally, lonely individuals had similar proportions of proxy informants while alive $(5.1 \%)$ compared with nonlonely individuals $(3.9 \% ; P=.14)$.

\section{Loneliness and Symptom Burden}

We first assessed the relationship between loneliness at EOL and individual symptoms. Compared with nonlonely decedents, a larger proportion of lonely decedents experienced each individual symptom; the most prevalent was pain $(69.1 \%$ vs $59.5 \%)$ (Figure 2$)$. In bivariate analyses, lonely decedents had increased odds of being troubled by pain of any severity $(P<.001)$, difficulty breathing $(P=.001)$, severe fatigue $(P=.02)$, difficulty controlling limbs $(P=.01)$, periodic confusion $(P<.001)$, and difficulty awakening $(P<.001)$ (Figure 3$)$.

We next examined the relationship between loneliness and total symptom burden. Loneliness was associated with increased total symptom burden in an unadjusted model $(P<.001)$ and remained significant after adjusting for demographics, socioeconomic status, and multimorbidity (model 1; $P<.001$ ); depressive symptoms (model 2; $P=.006$ ); family and friends (model $3 ; P<.001$ ); and social support (model 4; $P<.001$ ). The fully adjusted model included all covariates in models 1 to 4 and remained statistically significant (model 5, $P=.004$ ) (Table S1), indicating that loneliness was an independent predictor of total symptom burden at EOL.

\section{Loneliness and End-of-Life Care and Advance Care Planning}

Compared with nonlonely decedents, lonely decedents had greater odds of dying in a nursing home (adjusted odds ratio $[\mathrm{aOR}]=1.78 ; 95 \%$ confidence interval $[\mathrm{CI}]=1.30-2.42$; $P<.001)$ and using life support equipment in the last 2 years of life $(\mathrm{aOR}=1.36 ; 95 \% \mathrm{CI}=1.08-1.71 ; P=.01)($ Table 2$)$. However, loneliness was not associated with other measures of intense EOL care (eg, late hospice referral, ICU use, or dialysis use) or measures of advance care planning.

\section{DISCUSSION}

In this study of 2896 older decedents from a large nationally representative sample, we found that loneliness affected more than one-third of older adults near EOL. Lonely older adults were more likely to experience bothersome symptoms and were more likely to have a higher total symptom burden, even after controlling for confounders. Although lonely older adults had similar odds of advance care planning, they were exposed to more intense EOL care compared with nonlonely decedents. High symptom burden and exposure to intense or aggressive EOL care are major drivers of patient suffering and healthcare overuse. ${ }^{11,16}$ Thus our findings have important implications for lonely individuals' well-being, care quality, and healthcare costs at EOL.

Greater exposure to aggressive and painful interventions may be partially responsible for the increased symptom burden lonely individuals experience at EOL. ${ }^{17} \mathrm{We}$ observed that lonely individuals were more likely to receive life support at EOL; however, we did not observe an association between loneliness and other measures of aggressive care and healthcare overuse in the last 2 years of life, including ICU stays. Our findings are consistent with other studies that showed no difference in hospitalizations $^{22,35}$ among lonely vs nonlonely older adults. A study by Shaw and colleagues found that lonely individuals have lower Medicare expenditures compared with nonlonely individuals, some of which may be related to lower inpatient expenditures after adjusting for socioeconomic and health status. ${ }^{36}$ However, this study did not specifically evaluate changes in healthcare expenditures or utilization near death. Although it suggests that lonely individuals may be less likely to present for care in the years preceding death, it is possible loneliness may still be associated with more intense EOL care and higher expenditures near death.

Additionally, studies suggest that lonely older adults are more likely to experience emergent hospitalizations ${ }^{37}$ and subsequent rehospitalization. ${ }^{35}$ Increased acuity may necessitate more intense and costly interventions in emergent situations or near EOL. The broad EOL time period in our study may have prevented discernment of important differences that arise just proximal to death when use of life-sustaining measures like life support are more common. Future qualitative and ethnographic studies could better characterize these differences.

Our study also found that overall nursing home utilization (as indicated by number of nursing home nights) is similar for lonely and nonlonely individuals, which is supported by others using Medicare claims data. ${ }^{36}$ However, we also observed that lonely older adults have higher odds of dying in a nursing home, a troubling finding because it may further isolate lonely people from their communities and support systems, and worsen quality of life near death. However, this finding is not surprising given lonely individuals' propensity toward multimorbidity, cognitive decline, and poor physical functioning earlier in life. ${ }^{2,4-6}$ These health conditions, along with lower engagement in health-promoting behaviors, ${ }^{38,39}$ can place lonely individuals at risk for higher symptom burden throughout life, not just near death. Longitudinal studies could shed light on the onset and duration of symptom-related suffering for lonely older adults, informing interventions to improve quality of life in this population.

Our study has limitations. First, we used loneliness data from the most proximal HRS interview before death; these data may not have accurately depicted participants' EOL experience. However, prior studies have assessed loneliness as an exposure variable and found that its health effects can persist over time. ${ }^{2}$ Additionally, variability in loneliness over time would have biased our results toward the null.

Second, use of proxy-reported symptoms and EOL care may introduce recall bias but have shown moderate to good 
reliability when describing objectively observable symptoms and EOL care quality. ${ }^{40-42}$

Third, we lacked data on specific stigma-carrying diagnoses (eg, human immunodeficiency virus) that could exacerbate loneliness and lead to unmeasured confounding.

Fourth, there are challenges with current loneliness measurement tools that frequently treat loneliness as a disease state rather than a complex psychological and social phenomenon. However, the three-item UCLA Loneliness Scale is widely used and comparable with other national studies of loneliness, making it our best approximation of loneliness. Additionally, using a dichotomous measure for loneliness may have reduced statistical power, limiting ability to discern an effect if one did exist even though other studies have used this approach successfully.,22

Fifth, although we determined that loneliness did not predict why individuals may have lacked exit interviews (and hence were excluded from our study), more than 1700 decedents in the HRS were excluded for having incomplete or missing loneliness data. Whether these individuals were lonely or socially isolated is unclear. Finally, given the cross-sectional nature of our study, we cannot make conclusions about causality or exclude potential longitudinal interactions between other potential contributors to the EOL experience.

Our study also has strengths. First, to our knowledge, this is the first study to explore the association between loneliness, symptom burden, and intensity of care at EOL. Second, we used a large nationally representative sample, using survey weights. Third, we utilized multivariable models adjusting for multiple potential factors that could contribute to differences in EOL experience. Importantly, we controlled for measures of social support that may have impacted our outcomes of interest. Finally, we incorporated additional sensitivity analyses to assess for variability in loneliness variable over time and potential sources of recall and reporting bias.

Our findings have important implications for clinical practice and policy. Loneliness can have profound effects on symptom burden and quality of life near death. Thus we must be able to identify at-risk lonely patients ${ }^{43}$ near EOL as well as throughout the life continuum. Value-based repayment models to incentivize screening for loneliness in routine medical care have been proposed, ${ }^{7,44}$ and such tools can be particularly important as individuals near EOL when social networks become scarcer and community engagement wanes. ${ }^{10}$ Ultimately, after an initial loneliness screen, an important starting point is a candid conversation between patients and their providers to explore whether and how loneliness impacts their experiences, hopes, fears, and future care preferences, particularly near EOL. Some health systems are even forming partnerships with community-based groups targeting socially isolated and vulnerable older adults to further identify and mitigate loneliness among individuals who may have difficulty presenting for care and linking them with providers who can further support them. ${ }^{45}$

In addition to identifying at-risk lonely individuals near EOL through screening, unique interventions addressing the complex nature of loneliness at EOL are needed. Prior studies have indicated that targeting maladaptive thinking (eg, teaching ways to reframe one's thinking about social interactions, promoting positive coping, and managing social anxiety) may be the most effective intervention to address loneliness. ${ }^{1,46}$ Other interventions promoting social support, social access, and social skills training were also effective but to a lesser degree. ${ }^{1}$ Given this context, during the EOL period, interventions such as referrals to mental health providers or social workers to perform life review and engage in reflection around role or identify loss as a result of debility or disease could be useful. It may also include referral to social services or chaplaincy to help lonely individuals reconnect with loved ones, their faith, or spirituality near EOL. Where these connections are absent, volunteer-based community social support interventions have been helpful. ${ }^{47,48}$

In conclusion, we found that lonely older adults appear to have more symptom burden at EOL, are more likely to die in a nursing home, and are exposed to more intense EOL care that may contribute to more suffering near death. Screening for loneliness is thus important, particularly near EOL as disease burden mounts and social connections wane. Interdisciplinary interventions must target the complex psychological, social, existential, and health underpinnings related to loneliness. Future work to design and evaluate effective interventions to address the vulnerable EOL period is necessary.

\section{ACKNOWLEDGMENTS}

Financial Disclosure: The HRS is sponsored by the National Institute on Aging (NIA) (U01 AG009740) and is conducted at the Institute for Social Research, University of Michigan. Nauzley C. Abedini is supported by the National Clinician Scholars Program at the Institute for Healthcare Policy and Innovation. HwaJung Choi is supported by an NIA grant (K01AG057820). Melissa Y. Wei is supported by an NIA grant (K23AG056638). Kenneth M. Langa is supported by NIA grants (P30 AG053760 and P30 AG024824). Vineet Chopra is supported by grants from the Agency for Healthcare Research and Quality (1-R18-HS-025891-01), Department of Veterans Affairs HSR\&D (I01 HX001101-01), and Blue Cross Blue Shield of Michigan.

Conflict of Interest: The authors have declared no conflicts of interest for this article.

Author Contributions: Nauzley Abedini had full access to all of the data in the study and takes responsibility for the integrity of the data and the accuracy of the data analysis. Concept and design: All authors. Acquisition, analysis, or interpretation of data: Abedini and Choi. Drafting of the manuscript: Abedini. Critical revision of the manuscript for important intellectual content: All authors. Administrative, technical, or material support: Abedini and Chopra. Supervision: Chopra.

Sponsor's Role: No funder/sponsor had any role in the design and conduct of the study; collection, management, analysis, and interpretation of the data; preparation, review, or approval of the manuscript; and the decision to submit the manuscript for publication. 


\section{REFERENCES}

1. Masi CM, Chen HY, Hawkley LC, Cacioppo JT. A meta-analysis of interventions to reduce loneliness. Pers Soc Psychol Rev. 2011;15(3):219-266.

2. Perissinotto CM, Stijacic Cenzer I, Covinsky KE. Loneliness in older persons: a predictor of functional decline and death. Arch Intern Med. 2012;172(14): 1078-1083.

3. Wilson C, Moulton B. Loneliness among older adults: a national survey of adults 45+. Prepared by Knowledge Networks and Insight Policy Research. Washington, DC: AARP; 2010.

4. Cacioppo JT, Hawkley LC, Thisted RA. Perceived social isolation makes me sad: 5-year cross-lagged analyses of loneliness and depressive symptomatology in the Chicago Health, Aging, and Social Relations Study. Psychol Aging. 2010;25(2):453-463.

5. Cacioppo JT, Hawkley LC. Perceived social isolation and cognition. Trends Cogn Sci. 2009;13(10):447-454.

6. Rico-Uribe LA, Caballero FF, Martín-María N, Cabello M, AyusoMateos JL, Miret M. Association of loneliness with all-cause mortality: a meta-analysis. PLOS One. 2018;13(1):e0190033.

7. National Academies of Sciences, Engineering, and Sciences. The Health and Medical Dimensions of Social Isolation and Loneliness in Older Adults. https://www8.nationalacademies.org/pa/projectview.aspx?key=51322. 2019. Accessed January 12, 2019.

8. Centers for Medicare \& Medicaid Services. The Accountable Health Communities Health-Related Social Needs Screening Tool. 2019. https://innovation. cms.gov/Files/worksheets/ahcm-screeningtool.pdf. Accessed January 12, 2019.

9. Baernholdt M, Hinton I, Yan G, Rose K, Mattos M. Factors associated with quality of life in older adults in the United States. Qual Life Res. 2012;21(3): 527-534.

10. Gott M, Wiles J, Moeke-Maxwell T, et al. What is the role of community at the end of life for people dying in advanced age? A qualitative study with bereaved family carers. Palliat Med. 2018;32(1):268-275.

11. Lorenz KA, Rosenfeld K, Wenger N. Quality indicators for palliative and end-of-life care in vulnerable elders. J Am Geriatr Soc. 2007;55(suppl 2): S318-S326.

12. Smith AK, Cenzer IS, Knight SJ, et al. The epidemiology of pain during the last 2 years of life. Ann Intern Med. 2010;153(9):563-569.

13. Singer AE, Meeker D, Teno JM, Lynn J, Lunney JR, Lorenz KA. Symptom trends in the last year of life from 1998 to 2010: a cohort study. Ann Intern Med. 2015;162(3):175-183.

14. Patel KV, Guralnik JM, Phelan EA, et al. Symptom burden among community-dwelling older adults in the United States. J Am Geriatr Soc. 2019;67(2):223-231.

15. Hunt LJ, Smith AK. High symptom burden in older adults: a clarion call for geriatrics and palliative care research and training. J Am Geriatr Soc. 2019; 67(2):208-210.

16. Earle CC, Landrum MB, Souza JM, Neville BA, Weeks JC, Ayanian JZ. Aggressiveness of cancer care near the end of life: is it a quality-of-care issue? J Clin Oncol. 2008;26(23):3860-3866.

17. Wright AA, Keating NL, Ayanian JZ, et al. Family perspectives on aggressive cancer care near the end of life. JAMA. 2016;315(3):284-292.

18. Cacioppo JT, Cacioppo S. Loneliness in the modern age: an evolutionary theory of loneliness (ETL). Adv Exp Soc Psychol. 2018;58:127-197.

19. Health and Retirement Study, public use data set. Produced and distributed by the University of Michigan with funding from National Institute on Aging (grant NIA U01AG009740). https://hrs.isr.umich.edu/data-products/accessto-public-data. Accessed June 23, 2018.

20. Weir DR. Validating mortality ascertainment in the Health and Retirement Study. Survey Research Center. https://hrs.isr.umich.edu/sites/ default/files/biblio/Weir_mortality_ascertainment.pdf. 2016. Accessed August 12, 2018.

21. Smith J, Ryan L, Fisher GG, Sonnega A, Weir DR. Psychosocial and Lifestyle Questionnaire 2006-2016. Survey Research Center, Institute for Social Research. https:/hrs.isr.umich.edu/sites/default/files/biblio/HRS\%202006-2016\%20SAQ\% 20Documentation_07.06.17_0.pdf. 2017. Accessed August 12, 2018.

22. Gerst-Emerson K, Jayawardhana J. Loneliness as a public health issue: the impact of loneliness on health care utilization among older adults. Am J Public Health. 2015;105(5):1013-1019.

23. Hughes ME, Waite LJ, Hawkley LC, Cacioppo JT. A short scale for measuring loneliness in large surveys: results from two population-based studies. Res Aging. 2004;26(6):655-672.

24. Wallace R, Herzog A. Overview of the health measures in the Health and Retirement Study. J Hum Resour. 1995;30(suppl):S84-S107.

25. Klinkenberg M, Willems DL, van der Wal G, Deeg DJ. Symptom burden in the last week of life. J Pain Symptom Manage. 2004;27(1):5-13.

26. Basilevsky A. Statistical Factor Analysis and Related Methods: Theory and Applications. New York, NY: Wiley; 1994.
27. Earle CC, Park ER, Lai B, Weeks JC, Ayanian JZ, Block S. Identifying potential indicators of the quality of end-of-life cancer care from administrative data. J Clin Oncol. 2003;21(6):1133-1138.

28. Bischoff KE, Sudore R, Miao Y, Boscardin WJ, Smith AK. Advance care planning and the quality of end-of-life care in older adults. J Am Geriatr Soc. 2013;61(2):209-214.

29. Wei MY, Kabeto MU, Langa KM, Mukamal KJ. Multimorbidity and physical and cognitive function: performance of a new multimorbidity-weighted index. J Gerontol A Biol Sci Med Sci. 2018;73(2):225-232.

30. Wei MY, Mukamal KJ. Multimorbidity, mortality, and long-term physical functioning in 3 prospective cohorts of community-dwelling adults. Am J Epidemiol. 2018;187(1):103-112.

31. Wallace R, Herzog A, Ofstedal MB, Steffick D, Fonda S, Langa K. Documentation of Affective Functioning Measures in the Health and Retirement Study. Survey Research Center, University of Michigan. http://hrsonline.isr. umich.edu/sitedocs/userg/dr-005.pdf. 2000. Accessed August 12, 2018.

32. Cornwell EY, Waite LJ. Measuring social isolation among older adults using multiple indicators from the NSHAP study. J Gerontol B Psychol Sci Soc Sci. 2009;64(suppl 1):i38-i46.

33. Ofstedal MB, Weir DR, Chen K, Wagner J. Updates to HRS Sample Weights. Ann Arbor, MI: University of Michigan; 2011.

34. White IR, Royston P, Wood AM. Multiple imputation using chained equations: issues and guidance for practice. Stat Med. 2011;30:377-399.

35. Newall N, McArthur J, Menec VH. A longitudinal examination of social participation, loneliness, and use of physician and hospital services. J Aging Health. 2015;27(3):500-518.

36. Shaw JG, Farid M, Noel-Miller C, et al. Social isolation and Medicare spending: among older adults, objective social isolation increases expenditures while loneliness does not. J Aging Health. 2017;29(7):1119-1143.

37. Molloy GJ, McGee HM, O’Neill D, Conroy RM. Loneliness and emergency and planned hospitalizations in a community sample of older adults. J Am Geriatr Soc. 2010;58(8):1538-1541.

38. Schrempft S, Jackowska M, Hamer M, Steptoe A. Associations between social isolation, loneliness, and objective physical activity in older men and women. BMC Public Health. 2019;19(1):74.

39. Kobayashi LC, Steptoe A. Social isolation, loneliness, and health behaviors at older ages: longitudinal cohort study. Ann Behav Med. 2018;52(7):582-593.

40. Kutner JS, Bryant LL, Beaty BL, Fairclough DL. Symptom distress and quality-of-life assessment at the end of life: the role of proxy response. J Pain Symptom Manage. 2006;32(4):300-310.

41. Makaroun LK, Teno JM, Freedman VA, Kasper JD, Gozalo P, Mor V. Late transitions and bereaved family member perceptions of quality of end-of-life care. J Am Geriatr Soc. 2018;66(9):1730-1736.

42. McPherson CJ, Wilson KG, Lobchuk MM, Brajtman S. Family caregivers' assessment of symptoms in patients with advanced cancer: concordance with patients and factors affecting accuracy. J Pain Symptom Manage. 2008;35(1):70-82.

43. Murthy V. Work and the loneliness epidemic. Harvard Business Review. https://hbr.org/cover-story/2017/09/work-and-the-loneliness-epidemic. October 12, 2017. Accessed November11, 2018.

44. Perissinotto C, Holt-Lunstad J, Periyakoil VS, Covinsky K. A practical approach to assessing and mitigating loneliness and isolation in older adults. J Am Geriatr Soc. 2019;67(4):657-662.

45. Abedini NC, Solway E, Piette J, Malani P. Cross-Sector Collaborations to Decrease Loneliness and Social Isolation in Older Adults. Health Affairs Blog Web site. https://www.healthaffairs.org/do/10.1377/hblog20190618.629601/ full/. 2019. Accessed October 10, 2019.

46. Cacioppo S, Grippo AJ, London S, Goossens L, Cacioppo JT. Loneliness: clinical import and interventions. Perspect Psychol Sci. 2015;10(2):238-249.

47. McLoughlin K, Rhatigan J, McGilloway S, et al. INSPIRE (INvestigating social and PractIcal suppoRts at the End of Life): pilot randomised trial of a community social and practical support intervention for adults with lifelimiting illness. BMC Palliat Care. 2015;14:65.

48. Dodd S, Hill M, Ockenden N, et al. 'Being with' or 'doing for'? How the role of an end-of-life volunteer befriender can impact patient wellbeing: interviews from a multiple qualitative case study (ELSA). Support Care Cancer. 2018;26(9):3163-3172.

\section{SUPPORTING INFORMATION}

Additional Supporting Information may be found in the online version of this article.

Table S1: Multivariable regression models predicting total symptom burden ${ }^{\mathrm{a}}$ in the last year of life in lonely vs nonlonely decedents with imputed covariates $(n=2896)$. 